\title{
Termination of Idiopathic Sustained Monomorphic Ventricular Tachycardia by Synchronized Electrical Cardioversion during Pregnancy
}

\author{
Sungmin Lee
}

Department of Emergency Medicine, Chonnam National University Hospital, Gwangju, Korea

The most common cardiac complications detected during pregnancy are an arrhythmia. However, idiopathic continuous monomorphic ventricular tachycardia (VT) during pregnancy is unusual. A 31-year-old pregnant woman presented at 20 weeks of gestation with progressive palpitation and episodes of agitation. An initial 12-lead electrocardiogram (ECG) showed normal sinus rhythm. However, 30 minutes after presenting at the emergency room, she complained of chest pain. A subsequent ECG showed wide complex monomorphic VT. We attempted to administer an antiarrhythmic drug, but the patient refused any medication because of concerns regarding possible adverse effects on the fetus. Therefore, we performed synchronized electrical cardioversion eight times. After the eighth synchronized cardioversion at $200 \mathrm{~J}$, the ECG showed successful restoration of sinus rhythm. The condition of the fetus was monitored via ultrasonography and cardiotocography, and no adverse events were observed. We present the case of a successful synchronized electrical cardioversion performed in a woman at 20 weeks of gestation because of sustained symptomatic VT.

Key Words: electric countershock; pregnancy; tachycardia, ventricular

Arrhythmia is a common cardiac complication encountered during pregnancy [1]. The frequency of maternal arrhythmia is 1.2 per 1,000 pregnant women. And $50 \%$ of these women are asymptomatic. The frequency increases in the third trimester. However, severe arrhythmia requiring intensive therapies is rare. Particularly, ventricular tachycardia (VT) and ventricular fibrillation are rare during pregnancy, with a prevalence of 2 in 100,000 hospitalized women [2]. The precise mechanism underlying increased arrhythmia burden during pregnancy is unclear; however, the possible reason is a combination of hemodynamic, hormonal, and autonomic alterations during pregnancy. In general, the therapeutic approach to arrhythmia in pregnant women is similar to that in non-pregnant patients. Hyperthyroidism should be excluded, and other systemic disorders that can cause arrhythmia, such as pulmonary embolism, should be considered. An arrhythmia that leads to the development of either symptoms of hemodynamic compromise or posing a risk to the mother and fetus should be treated [3]. Cardioversion is necessary for the treatment of unmanageable arrhythmia and cardiopulmonary resuscitation. In spite of cardioversion is not contraindicated in pregnancy, its influence on fetal hemodynamics is uncertain [4]. Information on the effect of cardioversion on fetal health is scarce. Moreover, reports on successful synchronized electrical cardioversion during pregnancy are few [5]. Here, we present the case of synchronized electrical

Received on February 23, 2016 Revised on August 13, 2016 Accepted on September 1, 2016

Correspondence to: Sungmin Lee, Department of Emergency Medicine, Chonnam National University Hospital, 42 Jebong-ro, Dong-gu, Gwangju 61469, Korea Tel: +82-62-220-6809, Fax: +82-62-228-7417, E-mail: terran034@naver.com

*No potential conflict of interest relevant to this article was reported.

(cc) This is an Open Access article distributed under the terms of the Creative Commons Attribution Non-Commercial License (http://creativecommons.org/ licenses/by-nc/4.0/) which permits unrestricted non-commercial use, distribution, and reproduction in any medium, provided the original work is properly cited. Copyright (c) 2018 The Korean Society of Critical Care Medicine 
cardioversion performed during the second trimester of pregnancy without compromise of fetal hemodynamics.

\section{CASE REPORT}

A 31-year-old, previously healthy, pregnant woman presented at 20 weeks of gestation to her local hospital with progressive palpitations and agitation. The initial electrocardiogram (ECG) showed wide QRS tachycardia (184 beats/min), monomorphic left bundle branch block morphology in V1 lead, inferior axis (positive QRS in II, III, aVF lead. These features might suggest the idiopathic VT originating from right ventricular outflow tract) (Figure 1A). She was referred to our hospital for advanced cardiovascular treatment. On arrival, the patient's vital

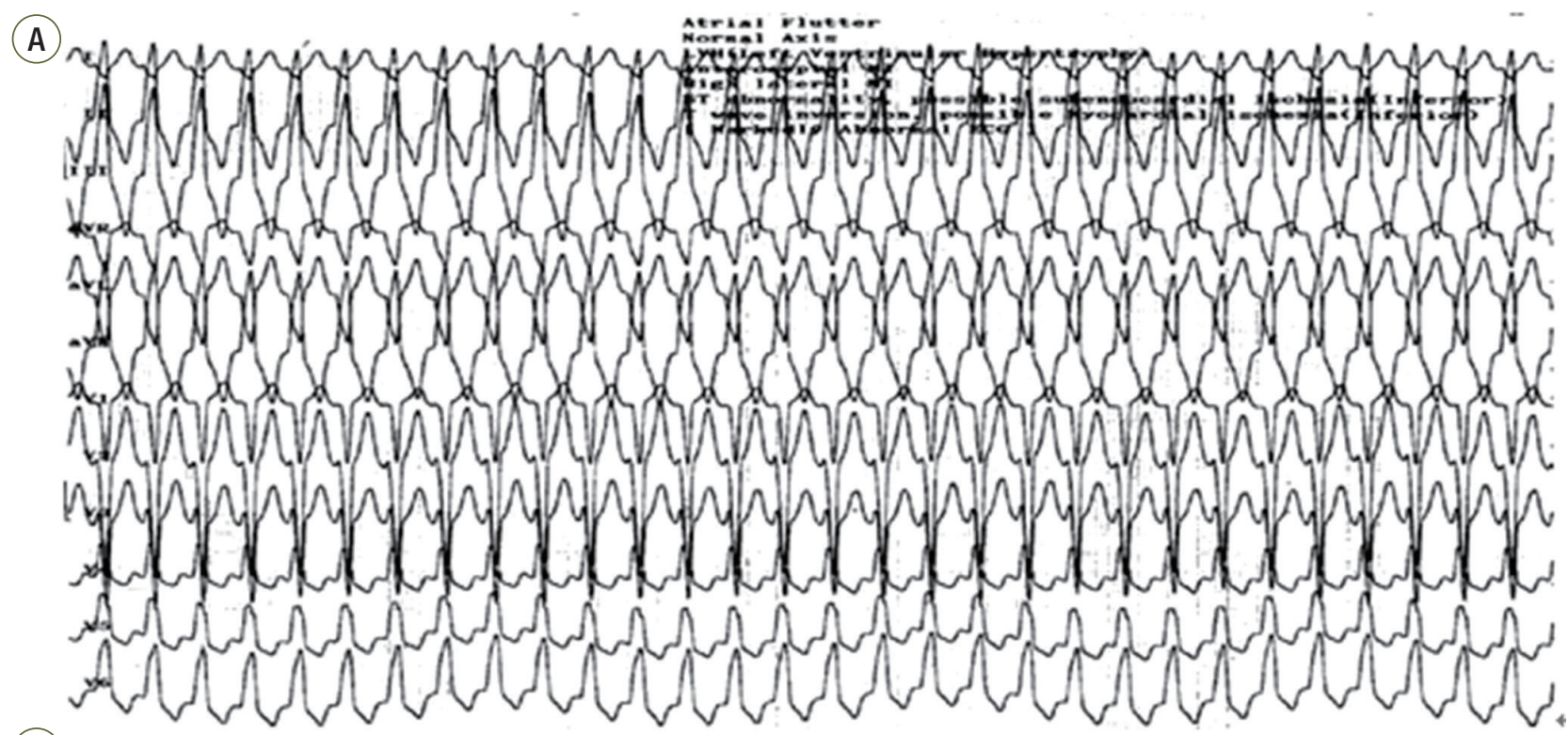

(B)

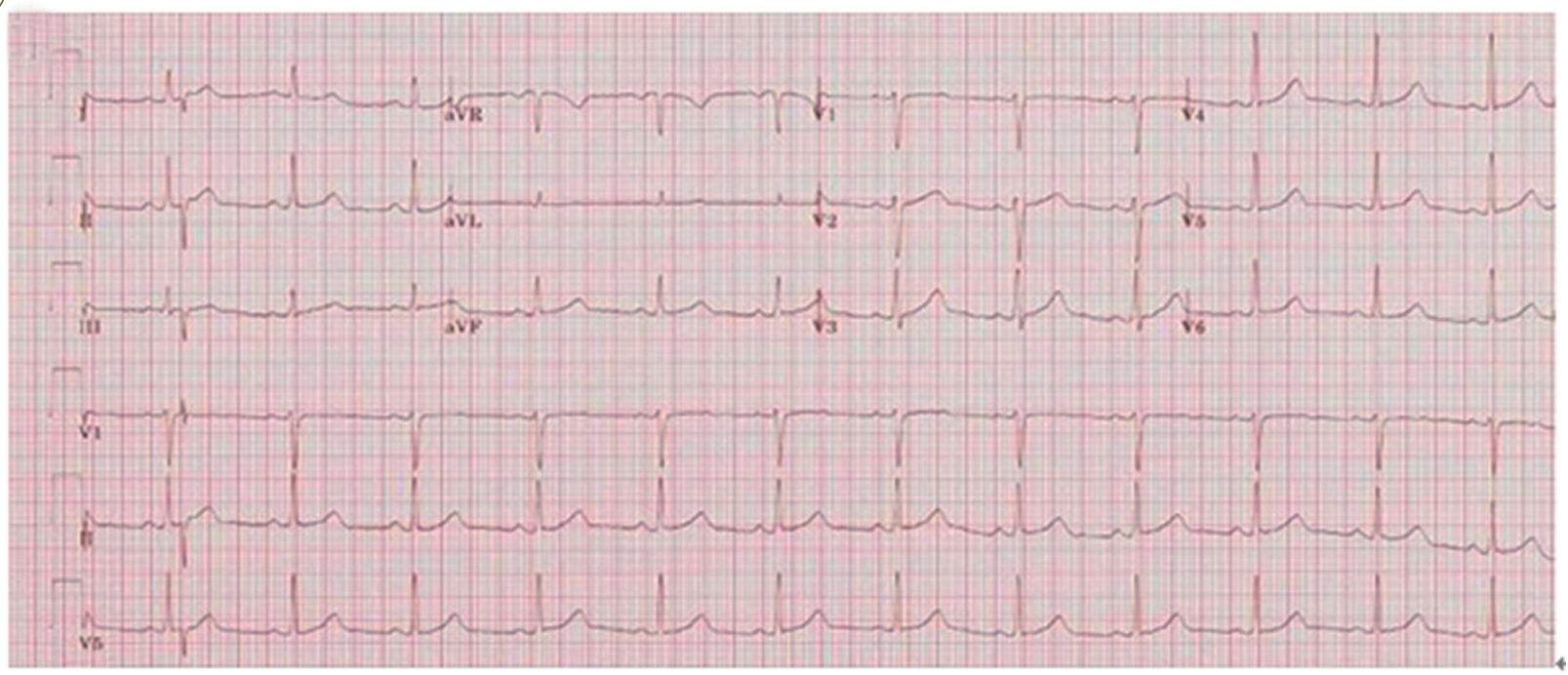

Figure 1. (A) Local 12-lead electrocardiogram (ECG) showing wide QRS tachycardia (184 beats/min), monomorphic left bundle branch block morphology in the V1 lead, inferior axis (positive QRS in leads II, III, aVF. These features suggest idiopathic ventricular tachycardia originating from the right ventricular outflow tract). (B) On arrival, 12-lead ECG showing normal sinus rhythm (73 beats/min) at the emergency room. 
signs were within the normal range. An initial 12-lead ECG showed normal sinus rhythm with a normal corrected QT interval (453 ms) (Figure 1B). However, 30 minutes after presenting to the emergency room, she complained of severe chest pain. A subsequent ECG showed wide complex monomorphic VT. At that time, the patient's blood pressure was 100/50 $\mathrm{mmHg}$. We attempted to administer of lidocaine or a beta-blocker; however, the patient refused any medication because of the concerns regarding possible adverse effect on the fetus. Therefore, we performed synchronized electrical cardioversion two times at $100 \mathrm{~J}$ (Figure 2A). Sinus conversion could not be achieved so, synchronized electrical cardioversion was performed at $150 \mathrm{~J}$ and $200 \mathrm{~J}$. For the first time, VT was terminated by seventh cardioversion at $200 \mathrm{~J}$. But, VT immediately recurred (Figure 2B). After the eighth synchronized cardioversion at $200 \mathrm{~J}$, the ECG showed sinus tachycardia (101 beats/min) (Figure 2C). We immediately consulted cardiologist and obstetrician. The obstetrician constantly monitored the fetal heart rate to ensure it was steady. The condition of the fetus was monitored via ultrasonography and cardiotocography, and no adverse events were observed. The cardiologist performed transthoracic echocardiography and thyroid function tests on

(A)

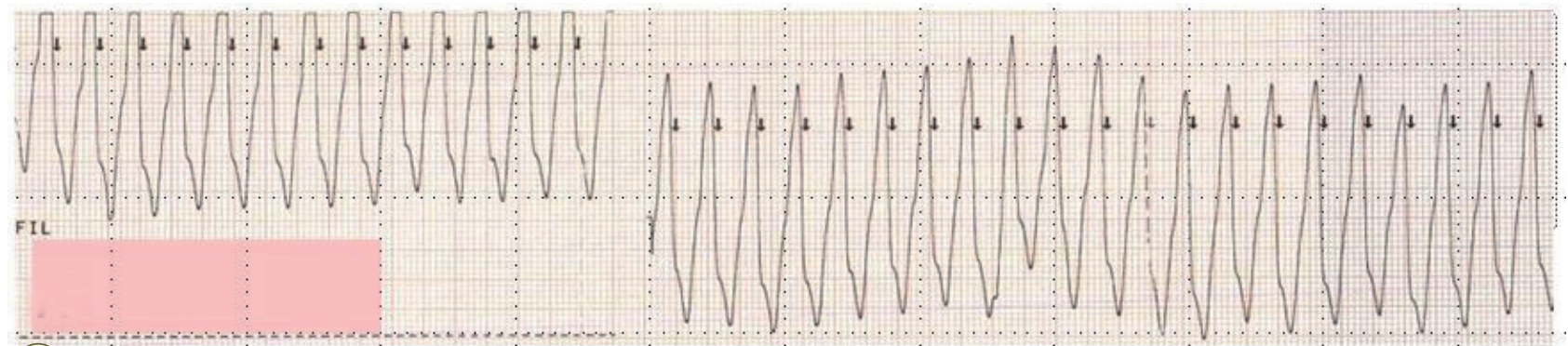

(B)
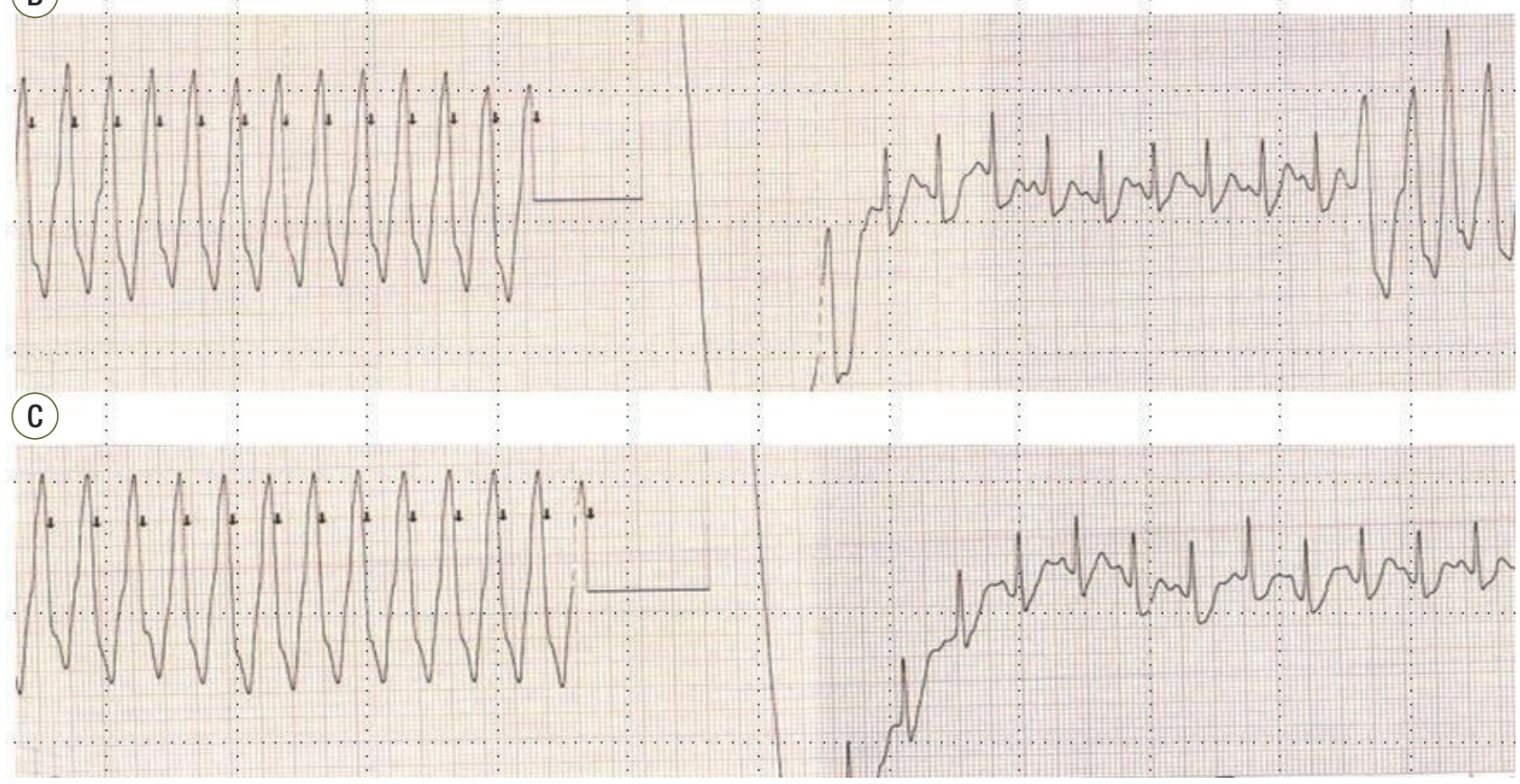

Figure 2. Continuous monitoring with 3-lead recordings obtained before, during, and after the synchronized direct-current cardioversion. (A) Ventricular tachycardia (VT) 30 minutes after the patient's arrival at the emergency room. (B) VT was terminated after the seventh cardioversion, after which it recurred. (C) Restoration of sinus rhythm after the eighth cardioversion. 
hospital day 1 , and normal results were obtained. The patient was admitted to the cardiac intensive care unit (CICU) for observation of the progress of the arrhythmia. Eventually, we persuaded her to take a beta-blocker (Selectol 200 mg; Pfizer Inc., New York, NY, USA) therapy once a day. While in the CICU, the patient did not show any symptoms of arrhythmia. She was transferred to a general ward 2 days later, and was discharged on hospital day 14 without complications. She successfully delivered a healthy girl of $2.7 \mathrm{~kg}$ at 37 weeks of gestation by cesarean section.

\section{DISCUSSION}

Although most cases of maternal arrhythmia are benign, some patients experience catastrophic events. Acute treatments for arrhythmia contain beta-blockers, calcium-channel blockers, lidocaine, and electrical cardioversion [6]. Patients with idiopathic VT are typically hemodynamically stable and idiopathic VT is associated with a good prognosis. Idiopathic VT may present for the first time during pregnancy [7]. It is often catecholamine sensitive, and treatment of outflow tract VT with cardioselective beta-blockers in pregnant patients is usually effective. Sotalol or flecainide may be considered where patients have severe symptoms that who fail to respond to beta-blocker therapy $[8,9]$. Lastly, cardioversion can be considered for VT that is refractory to other treatments.

Surely, mother and fetus are at risk of harm when VT appears during pregnancy. However, information on the impact of cardioversion on fetal health is limited. Also, obstetric cases of cardioversion have been rarely documented. But, synchronized direct cardioversion may not considerably affect the fetal health, because the mammalian fetal myocardium has a high fibrillation threshold, and the density of the current reaching the uterus is considerably low $[10,11]$. The decision about the timing of electrical cardioversion may be burdensome because of potentially adverse priorities between mother and fetus. However, synchronous direct electrical cardioversion should be performed immediately for severe maternal arrhythmia that does not comply with other therapies (including medical therapy), according to current recommendations. Electrical cardioversion is a reasonable option at all stages of pregnancy when arrhythmia is associated with hemodynamic instability [12]. Electrical cardioversion can be selected for drug refractory arrhythmia as it does not compromise blood flow to the fetus [4]. Moreover, because only a small amount of current reaches the fetus, the risk of induction of fetal arrhythmia is low [11]. However, in the later stages of pregnancy, a theoretical risk of initiation of preterm labor exists. Cases of emergency cesarean delivery because of fetal arrhythmia after cardioversion have been reported, and hence, fetal monitoring is advised [13].

The therapy points to protect the mother and the fetus through to delivery, after which chronic or definitive therapy can be conducted. However, our experience shows that electrical cardioversion is effective and safe during the second trimester of pregnancy without compromise to fetal hemodynamics. Indeed, the patient in the present case had normal fetal outcomes. Treatment of patients with gestational cardiac arrhythmias requires significant modifications to standard arrhythmia therapy. In some cases, cardioversion is the therapy of first choice, before drug therapy is used.

\section{REFERENCES}

1. Siu SC, Sermer M, Colman JM, Alvarez AN, Mercier LA, Morton BC, et al. Prospective multicenter study of pregnancy outcomes in women with heart disease. Circulation 2001;104:515-21.

2. Li JM, Nguyen C, Joglar JA, Hamdan MH, Page RL. Frequency and outcome of arrhythmias complicating admission during pregnancy: experience from a high-volume and ethnically-diverse obstetric service. Clin Cardiol 2008;31:538-41.

3. Enriquez AD, Economy KE, Tedrow UB. Contemporary management of arrhythmias during pregnancy. 
Circ Arrhythm Electrophysiol 2014;7:961-7.

4. Wang YC, Chen CH, Su HY, Yu MH. The impact of maternal cardioversion on fetal haemodynmics. Eur J Obstet Gynecol Reprod Biol 2006;126:268-9.

5. Tromp CH, Nanne AC, Pernet PJ, Tukkie R, Bolte AC. Electrical cardioversion during pregnancy: safe or not? Neth Heart J 2011;19:134-6.

6. Kron J, Conti JB. Arrhythmias in the pregnant patient: current concepts in evaluation and management. J Interv Card Electrophysiol 2007;19:95-107.

7. Lemery R, Brugada P, Bella PD, Dugernier T, van den Dool A, Wellens HJ. Nonischemic ventricular tachycardia: clinical course and long-term followup in patients without clinically overt heart disease. Circulation 1989;79:990-9.

8. Brodsky M, Doria R, Allen B, Sato D, Thomas G,
Sada M. New-onset ventricular tachycardia during pregnancy. Am Heart J 1992;123(4 Pt 1):933-41.

9. Chandra NC, Gates EA, Thamer M. Conservative treatment of paroxysmal ventricular tachycardia during pregnancy. Clin Cardiol 1991;14:347-50.

10. Rosemonda RL. Cardioversion during pregnancy. JAMA 1993;269:3167.

11. Page RL. Treatment of arrhythmias during pregnancy. Am Heart J 1995;130:871-6.

12. Cox JL, Gardner MJ. Treatment of cardiac arrhythmias during pregnancy. Prog Cardiovasc Dis 1993;36:137-8.

13. Barnes EJ, Eben F, Patterson D. Direct current cardioversion during pregnancy should be performed with facilities available for fetal monitoring and emergency caesarean section. BJOG 2002;109:1406-7. 